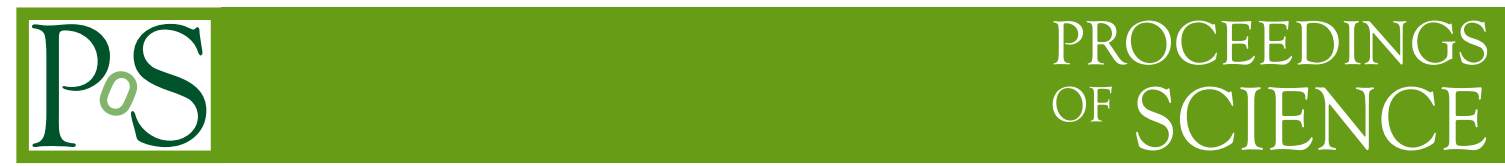

\title{
Prospects of LFV studies at Belle II
}

\author{
Dmitri Liventsev* (for the Belle II collaboration) \\ Virginia tech \\ Blacksburg, VA, USA \\ KEK \\ Tsukuba, Japan \\ E-mail: dmitri.liventsev@kek.jp
}

Lepton Flavour Violating (LFV) $\tau$ lepton decays are strongly suppressed in the Standard Model but some New Physics models predict the branching fractions of decays such as $\tau \rightarrow \ell \ell \ell$ and $\tau \rightarrow \mu \nu$ in the experimentally accesible range of $10^{-9}$ to $10^{-7}$. Belle has studied 48 different LFV $\tau$ lepton modes and set $90 \%$ C.L. upper limits of $\mathscr{O}\left(10^{-8}\right)$ on the branching fractions. In this paper we review these studies and discuss prospects of LFV searches in $\tau$ lepton decays at future Belle II experiment which should collect 50 times higher statistics than Belle.

The 19th International Workshop on Neutrinos from Accelerators-NUFACT2017

25-30 September, 2017

Uppsala University, Uppsala, Sweden

${ }^{*}$ Speaker. 
Table 1: Predictions of $\tau$ LFV decay modes in different models (from [10] and [11]).

\begin{tabular}{|c|c|c|c|c|}
\hline \hline & SUSY + GUT & Higgs mediated & Little Higgs & Non-universal $Z^{\prime}$ \\
\hline$\frac{\mathscr{B}(\tau \rightarrow \mu \mu \mu)}{\mathscr{B}(\rightarrow \mu \gamma)}$ & $\sim 2 \times 10^{-3}$ & $0.06-0.1$ & $0.4-2.3$ & $\sim 16$ \\
\hline$\frac{\mathscr{B}(\tau \rightarrow \mu e e)}{\mathscr{B}(\rightarrow \mu \gamma)}$ & $\sim 1 \times 10^{-2}$ & $\sim 1 \times 10^{-2}$ & $0.3-1.6$ & $\sim 16$ \\
\hline $\mathscr{B}(\rightarrow \mu \gamma)_{\max }$ & $<10^{-7}$ & $<10^{-10}$ & $<10^{-10}$ & $<10^{-9}$ \\
\hline \hline
\end{tabular}

\section{Introduction}

Lepton flavor violation (LFV) in charged lepton decays is forbidden in the Standard Model (SM) and highly suppressed even if neutrino mixing is taken into account, e.g. $\mathrm{B}(\tau \rightarrow \mu \gamma) \simeq$ $\mathrm{O}\left(10^{-40}\right)$ and $\mathrm{B}(\tau \rightarrow \mu \mu \mu) \simeq \mathrm{O}\left(10^{-14}\right)$ [1], which is inaccessible in current experiments. On the other hand, New Physics (NP) extensions of the SM, such as supersymmetry, leptoquark and many other models $[2,3,4,5,6,7,8,9]$ predict enhanced LFV decays with branching fractions as large as $10^{-8}$. The observation of a LFV $\tau$ decay would be an unambiguous sign of physics beyond the SM. $B$-factories Belle and BaBar and their successor Belle II can probe the entire range of LFV $\tau$ decays, i.e., $\tau \rightarrow \ell \gamma, \tau \rightarrow \ell \ell \ell, \tau \rightarrow \ell h h^{\prime}$ etc. This is particularly interesting as different NP scenarios predict very different patterns of $\tau$ LFV (Table 1 ). By observing a specific pattern of LFV $\tau$ decays, one can not only conclude that there is physics beyond the SM but also draw conclusions on the specific underlying NP scenario.

\section{The Belle and Belle II experiments}

The results presented here are based on a data sample collected at the $\Upsilon(4 S)$ resonance with the Belle detector operating at the KEKB asymmetric-energy $e^{+} e^{-}$collider [12].

The Belle detector is a large-solid-angle magnetic spectrometer that consists of a silicon vertex detector (SVD), a 50-layer central drift chamber (CDC), an array of aerogel threshold Cherenkov counters (ACC), a barrel-like arrangement of time-of-flight scintillation counters (TOF), and an electromagnetic calorimeter comprised of $\mathrm{CsI}(\mathrm{Tl})$ crystals (ECL) located inside a superconducting solenoid coil that provides a $1.5 \mathrm{~T}$ magnetic field. An iron flux return located outside the coil is instrumented to detect $K_{L}^{0}$ mesons and to identify muons (KLM). The detector is described in detail elsewhere [13].

Tracking at Belle is done using the SVD and CDC. Two configurations of the SVD, referred to as SVD1 and SVD2, were used; $140 \mathrm{fb}^{-1}$ were accumulated with SVD1 and the remainder with SVD2. SVD1 consists of three layers of double-sided silicon strip detectors arranged in a barrel surrounding a $20 \mathrm{~mm}$ beam pipe at radii of $30 \mathrm{~mm}, 45.5 \mathrm{~mm}$ and $60.5 \mathrm{~mm}$. SVD2 has a 4-layer configuration with radii of $20 \mathrm{~mm}, 43.5 \mathrm{~mm}, 70 \mathrm{~mm}$ and $80 \mathrm{~mm}$ surrounding a $15 \mathrm{~mm}$ radius beam pipe. The inner and outer radii of the CDC are $83 \mathrm{~mm}$ and $880 \mathrm{~mm}$.

Belle operated from 1999 to 2010 and accumulated an integrated luminosity of about $711 \mathrm{fb}^{-1}$ on the $\Upsilon(4 S)$ resonance. $\tau$ leptons are produced in $e^{+} e^{-} \rightarrow \tau^{+} \tau^{-}$process with a cross-section of $0.93 \mathrm{nb}$. Including data sets taken at other energies, there are about 0.9 billion $\tau^{+} \tau^{-}$events in the Belle data. 
The Belle II experiment at the asymmetric $e^{+} e^{-}$SuperKEKB collider [14] will start its operation in 2018. The designed luminosity of the SuperKEKB is $8 \times 10^{35} \mathrm{~cm}^{-2} \mathrm{~s}^{-1}$, which is 40 times higher than that of KEKB. The expected integrated luminosity of the Belle II is $50 \mathrm{ab}^{-1}$, which is 50 times higher than that of the Belle.

In order to achieve the designed luminosity, a new scheme based on the nano-beam is proposed by P. Raimondi [15]. The reduced beam size is achieved by decreasing the beam vertical beta function at the interaction point by $1 / 20$ as compared to KEKB and by increasing the beam current by a factor of two, while keeping the same beam parameters. The luminosity of this rate will also result in much higher background rates, leading to greater detector occupancy and radiation damage.

The Belle II detector is constructed reusing many parts of the Belle detector. Its main design goal is to maintain or improve the data quality with respect to Belle despite the increase of the event rate and the backgrounds by about an order of magnitude. To handle the high luminosity condition of SuperKEKB, the upgrade of detector system and its electronics are in progress. The most challenging experimental requirement is the detection of the decay point of the short-living $B$-mesons, relying on a high-performance vertex detector. Therefore, the first sub-detector is placed very close to the interaction point and surrounding the beam-pipe are the upgraded silicon vertex detectors, including two pixel layers (PXD) based on DEPFET sensors and four layers of double-sided silicon vertex detector (SVD). Next detector system is a large central drift chamber (CDC) which will provide tracking with precise momentum measurements as well as clear particle identification (PID) through the measurement of ionization energy loss. The CDC is surrounded by the upgraded barrel PID system, which consists of an imaging time-of-propagation counter (TOP). A focusing aerogel ring imaging Cherenkov detector (ARICH) provides PID measurements in the endcap region. The electromagnetic calorimeter (ECL) has been equipped with upgraded electronics to suppress the much higher beam-related backgrounds expected at SuperKEKB. The barrel region of the $K_{L}$ and muon detector (KLM) consists of resistive plate chambers. The innermost layers of the barrel region is made up of plastic scintillators to cope with the high neutron flux both from the interaction point and from the beam line. A detailed description of the baseline design can be found in the Technical Design Report [16].

\section{3. $\tau$ LFV Searches at Belle}

\subsection{Reconstruction of $\tau$ decays}

The search for a LFV $\tau$ lepton decay mode at Belle proceeds as follows: We select lowmultiplicity (two or four charged tracks) events with zero net charge. The candidate $e^{+} e^{-} \rightarrow$ $\tau^{+} \tau^{-}$event is then divided into hemispheres (signal and tag side) using the thrust axis in the c.m. frame (Fig. 1). Each hemisphere is considered as a possible candidate for the LFV decay under consideration. In the opposite hemisphere 1-prong $\tau$ decays ( $\tau \rightarrow \ell v v$ and $\tau \rightarrow h v$, covering about $85 \%$ of the $\tau$ decay width) are reconstructed (tag side). While these requirements remove $B \bar{B}$ and hadronic continuum events, a significant amount of Bhabha, muon-pair and two-photon processes remains. Also generic $\tau^{+} \tau^{-}$events can contribute to the background. Unlike the SM $\tau$ decays which have at least one neutrino, the LFV decay products have no undetectable particles, and one 
can apply tight selection criteria on the c.m. frame energy $E_{X}$ and invariant mass $M_{X}$ of the decay products. For signal, $E_{X}$ is approximately equal to the beam energy in the c.m. frame and $M_{X}$ peaks at the $\tau$ mass. Hence, we use a two-dimensional signal region in the $M_{X}$ vs. $\delta E=E_{\text {beam }}-E_{X}$ plane (Fig. 2). The size of the signal ellipse and other selection criteria are optimized using Monte Carlo (MC) simulation of the signal and the backgrounds (blind analysis). The residual backgrounds are estimated from sideband data or MC simulation.

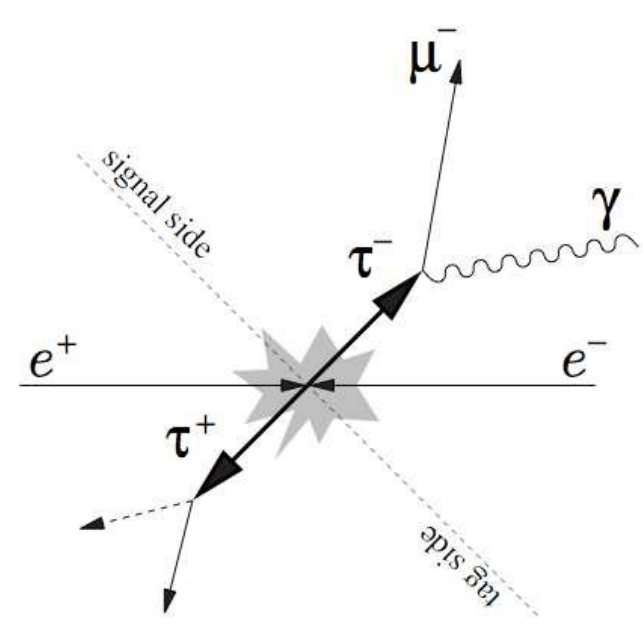

Figure 1: Illustration of the $e^{+} e^{-} \rightarrow \tau^{+} \tau^{-}$topology.

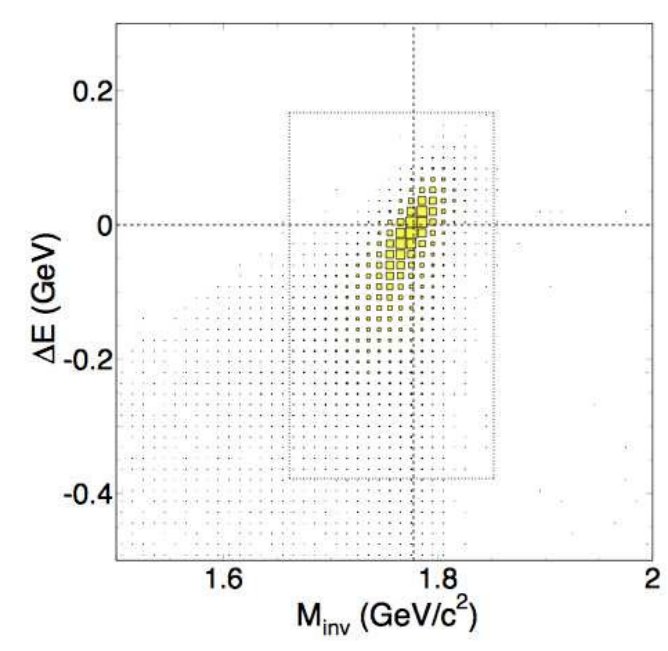

Figure 2: Search for $\tau \rightarrow \mu \gamma$ : The signal distribution of the invariant $\mu \gamma$ mass vs. $\Delta E=E_{\text {beam }}-E_{\mu \gamma}$ is shown.

For further background reduction, we introduce kinematic constraints. For example, we apply a requirement on the relation between the missing momentum $P_{\text {miss }}^{2}$ and the missing mass-squared $M_{\text {miss }}$ for various $\tau$ LFV analyses. This requirement removes $98 \%$ of the background from generic $\tau$ pairs and $80-90 \%$ of the radiative Bhabha, muon-pair and continuum events, while retaining $76 \%$ of the signal events in the $\tau \rightarrow \mu \gamma$ analysis.

Typical values of signal efficiency are between 3\% to $10 \%$, depending on the mode under study. This overall efficiency is the product of the following contributions: trigger (90\%), acceptance/reconstruction (70\%), charged-particle topology selection (70\%), particle identification (50\%), additional background reduction (50\%), $M_{X}$ vs. $\Delta E$ signal region requirement (50\%).

The evaluation of the upper limit is based on the prescription of Feldman-Cousins and its extensions to include systematic errors [17].

\section{$3.2 \tau \rightarrow \ell \gamma$}

The $\tau \rightarrow \mu \gamma$ mode suffers from backgrounds which mimic the signal. The dominant background remaining after applying all selections originates from $\tau^{+} \tau^{-}$events with initial state radiation, $\tau^{+} \tau^{-} \gamma$, where one of the $\tau$ s decays into $\mu \nu v$. Multi-photon radiative muon pairs are the second largest source of background: one of the two leptons and a radiated photon form a signal candidate, while the other lepton satisfies the selection for the tag side. In the current Belle analysis based on $545 \mathrm{fb}^{-1}$ [18], these backgrounds are studied using real data and MC simulation. After 
unblinding, 94 events are found in the $5 \sigma$ signal ellipse while expecting $88 \pm 7$ background events and a $90 \%$ C.L. upper limit of $4.5 \times 10^{-8}$ is set on the $\tau \rightarrow \mu \gamma$ branching fraction. The same procedure with minor modifications is used to study $\tau \rightarrow e \gamma$ decay. 55 events are found while $43 \pm 4$ expected and a $90 \%$ C.L. upper limit of $12.0 \times 10^{-8}$ is set.

\section{3 $\tau \rightarrow \ell \ell \ell$ and $\tau \rightarrow \ell V^{0}$}

The $\tau$ decay to three charged leptons is the cleanest search mode and backgrounds are at a negligible level in the Belle analysis. The analysis [19] is based on an integrated luminosity of $782 \mathrm{fb}^{-1}$ and six modes ( $\tau \rightarrow e e e, \mu \mu \mu, e \mu \mu, \mu e e, e \mu \mu, \mu e e$ ) are searched for. Due to the good performance of the lepton identification at Belle, all six modes are virtually background-free and no events are observed in the six signal ellipses. Upper limits on the branching fractions (at 90\% C.L.) between $1.5 \times 10^{-8}$ and $2.7 \times 10^{-8}$ are set. Similarly, $\tau$ modes to one lepton plus one hadron are very clean. The Belle search for $\tau \rightarrow \ell V^{0}$ (where $V^{0}$ stands for $\rho, \phi, \omega, K^{* 0}$ and $K^{* 0}$ ) [20] is based on $845 \mathrm{fb}^{-1}$ of data. The vector mesons are reconstructed on the signal side from two to three decay daughters and their invariant mass is required to be consistent with the mother particle. Ten modes are searched for $\left(\tau \rightarrow \mu \rho, e \rho, \mu \phi, e \phi, \mu \omega, e \omega, \mu K^{* 0}, e K^{* 0}, \mu K^{* 0}, e K^{* 0}\right.$ ) with dominant backgrounds coming from hadronic $\tau$ decay modes, hadronic continuum ( $\mu$ modes) and QED twophoton events ( $e$ modes). No events are seen in the signal ellipses except for $\mu \phi$ and $\mu K^{* 0}$ where one back-ground event is found in each mode. Upper limits (at $90 \%$ C.L.) between $1.2 \times 10^{-8}$ and $8.4 \times 10^{-8}$ are set on the branching fractions of the ten search modes.

\section{$3.4 \tau \rightarrow \ell h h^{\prime}$}

With some effort in background rejection, these modes may be still made clean. Belle published a search for eight lepton flavour violating $\left(\tau^{-} \rightarrow \ell^{-} h^{+} h^{-}, h, h^{\prime}=\pi, K\right)$ and six lepton number violating modes $\left(\tau^{-} \rightarrow \ell^{+} h^{-} h^{\prime-}, h, h^{\prime}=\pi, K\right)$ based on $854 \mathrm{fb}^{-1}$ of data [21]. Backgrounds come from generic hadronic $\tau$ decays where one or more particles are misidentified, so that $M_{\ell h h^{\prime}}$ and $\Delta E$ are within the signal ellipse despite the missing neutrino.

These backgrounds are suppressed with the following requirements: In the $\tau \rightarrow \mu \pi K$ mode a two-dimensional selection in the $M_{m i s s}^{2}$ vs. $P_{\text {miss }}$ plane is applied, which rejects $75 \%$ of the background while retaining about $75 \%$ of the signal. In the other modes, a requirement on $M_{\text {miss }}^{2}$ only is applied, which is about $90 \%$ efficient and rejects $50 \%$ of the remaining background. In the $\tau \rightarrow \mu \pi K$ search, the decay $\tau \rightarrow \pi \pi \pi \nu$ is explicitly vetoed by assigning the pion masses to all three charged particles on the signal side and recalculating the invariant mass. The invariant mass with the threefold pion mass hypothesis must be larger than $1.52 \mathrm{GeV} / c^{2}$, which rejects $65 \%$ of the background remaining at this point in the analysis and keeps $65 \%$ of the $\tau \rightarrow \mu \pi K$ signal. After unblinding the real data, no events are seen in the signal ellipses (except one event in each the $\tau^{-} \rightarrow \mu^{+} \pi^{-} \pi^{-}$and the $\tau^{-} \rightarrow \mu^{-} \pi^{+} K^{-}$channels) and $90 \%$ C.L. upper limits between $2.0 \times 10^{-8}$ and $8.6 \times 10^{-8}$ are set on the corresponding branching fractions.

The current results from the $B$ factory experiments for various LFV $\tau$ decays are summarized in Fig. 3. We have searched for 48 LFV $\tau$ decays and in all modes, the sensitivity has been improved by two orders of magnitude compared to the CLEO experiment results. Upper limits for the branching fractions are at the $10^{-8}$ level, approaching regions sensitive to New Physics. 


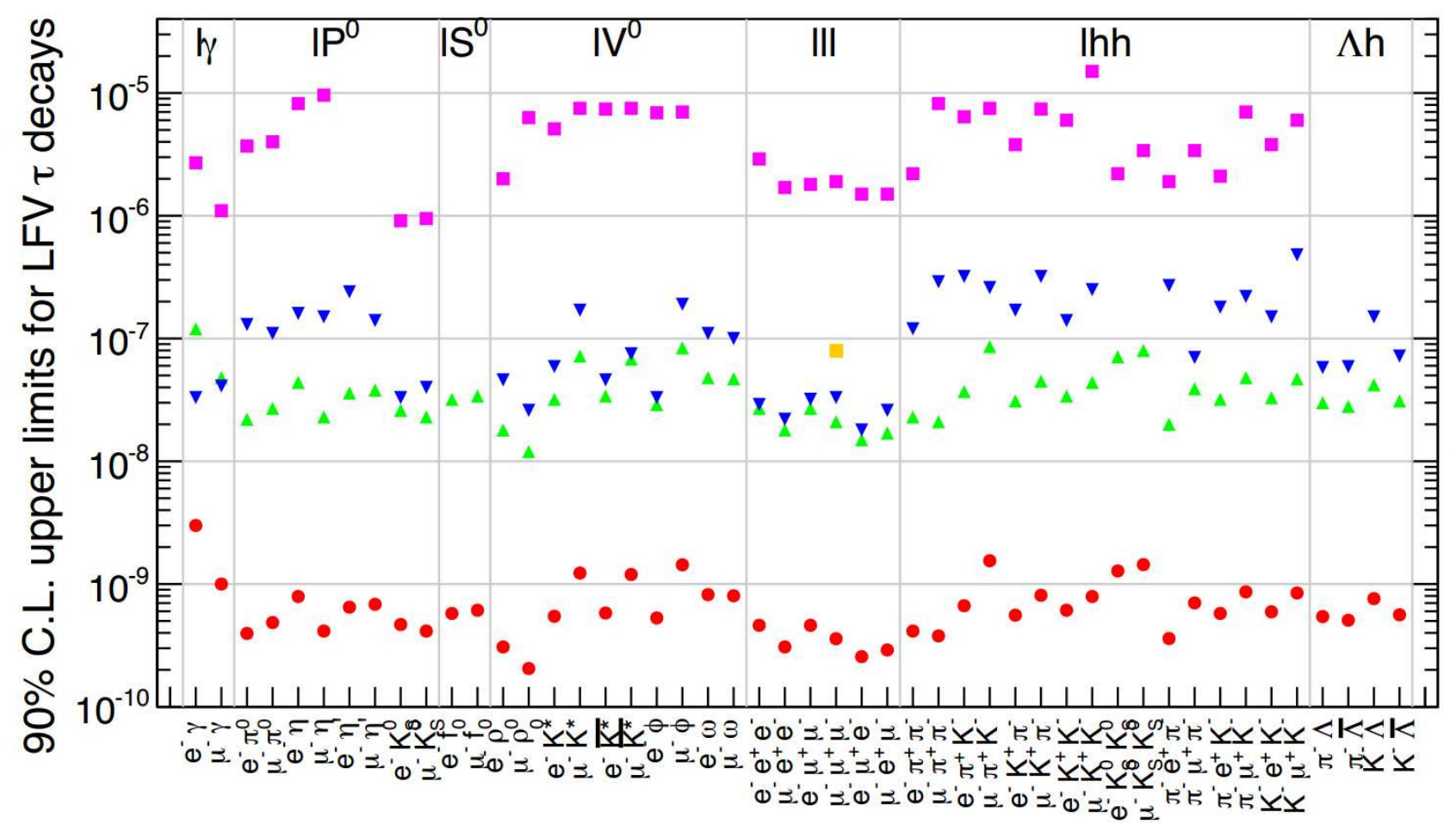

Figure 3: Summary of the $B$ factory searches for LFV tau lepton decay modes. Also $90 \%$ C.L. upper limits expected at Belle II are shown [22].

\section{Prospects for $\tau$ LFV Searches at Belle II}

With a data sample of $50 \mathrm{ab}^{-1}$ accumulated at SuperKEKB, the number of $\tau^{+} \tau^{-}$events will increase to $N_{\tau \tau}=5 \times 10^{10}$. For $\tau \rightarrow \ell \ell \ell$ and $\tau \rightarrow \ell h^{0}$ backgrounds are negligible up to several $\mathrm{ab}^{-1}$ and the corresponding upper limits change proportional to $1 / N_{\tau \tau}$. As background candidates appear, the dependence gradually changes to $1 / \sqrt{N_{\tau \tau}}$. For $\tau \rightarrow \ell \gamma$, backgrounds are not negligible and the improvement of the upper limit is proportional to $1 / \sqrt{N_{\tau \tau}}$ only. For this mode, the resolution in the discriminant variables for signal $\left(M_{X}\right.$ and $\left.\Delta E\right)$ at Belle II and an accurate prediction of the background will play an important role. There is also potential for improving the kinematic selection of the signal. The projected upper limits for all modes studied at Belle at the end of Belle II assuming current background conditions are shown in Fig. 3. If the current signal-to-background conditions can be maintained, Belle II can probe the branching fractions of these decay modes at the level of $10^{-9}-10^{-10}$.

\section{References}

[1] X.-Y. Pham, Eur. Phys. J. C 8, 513 (1999).

[2] A. Ilakovac, Phys. Rev. D 62, 036010 (2000).

[3] D. Black et al., Phys. Rev. D 66, 053002 (2002).

[4] C.-H. Chen and C.-Q. Geng, Phys. Rev. D 74, 035010 (2006). 
[5] E. Arganda, M.J. Herrero and J. Portoles, JHEP 0806, 079 (2008).

[6] R. Benbrik and C. H. Chen, Phys. Lett. B 672, 172 (2009).

[7] Z. H. Li, Y. Li and H. X. Xu, Phys. Lett. B 677, 150 (2009).

[8] W. J. P. Li et al., Int. J. Mod. Phys. A 25, 4827 (2010).

[9] W. Liu, C. X. Yue and J. Zhang, Eur. Phys. J. C 68, 197 (2010).

[10] M. Blanke, A. J. Buras, B. Duling, A. Poschenrieder and C. Tarantino, JHEP 0705 (2007) 013 [hep-ph/0702136].

[11] C. Yue, Y. Zhang, L. Liu, Phys. Lett. B 547 (2002) 252.

[12] S. Kurokawa and E. Kikutani, Nucl. Instr. and Meth. A 499, 1 (2003), and other papers included in this Volume.

[13] A. Abashian et al. (Belle Collaboration), Nucl. Instr. and Meth. A 479, 117 (2002).

[14] Y. Morita, K. Akai, T. Furuya, A. Kabe, S. Mitsunobu, M. Nishiwaki and S. Takano, Conf. Proc. C 100523, TUPEB011 (2010).

[15] C. Milardi et al. 2009 ICFA Beam Dyn. Newslett 48 23-33 (2009).

[16] T. Abe et al. [Belle II Collaboration], arXiv:1011.0352 [physics.ins-det].

[17] G. J. Feldman and R. D. Cousins, Phys. Rev. D 57 (1998) 3873 arXiv:physics/9711021 [physics.data-an].

[18] K. Hayasaka et al. [Belle Collaboration], Phys. Lett. B 666 (2008) 16 arXiv:0705.0650 [hep-ex].

[19] K. Hayasaka, K. Inami, Y. Miyazaki, K. Arinstein, V. Aulchenko, T. Aushev, A. M. Bakich and A. Bay et al. [Belle Collaboration], Phys. Lett. B 687 (2010) 139 arXiv:1001.3221 [hep-ex].

[20] Y. Miyazaki et al. [Belle Collaboration], Phys. Lett. B 699 (2011) 251 arXiv:1101.0755 [hep-ex].

[21] Y. Miyazaki et al. [Belle Collaboration], Phys. Lett. B 719 (2013) 346 arXiv:1206.5595 [hep-ex].

[22] B. Moore, BELLE2-THESIS-2017-002. 